\title{
Prevalence of human papillomavirus types 16 and 18 in penile carcinoma: a study of 41 cases using PCR
}

\author{
K W Chan, K Y Lam, A C L Chan, P Lau, G Srivastava
}

\begin{abstract}
Aims-To determine retrospectively the prevalence of human papillomavirus (HPV) types 16 and 18 in penile carcinomas.

Methods-Forty one surgically resected penile carcinomas from the archives at Queen Mary Hospital, Hong Kong, were reviewed and classified into verrucous carcinoma, and well, moderately, and poorly differentiated squamous cell carcinomas. Paraffin wax embedded tumour tissue was sectioned and analysed for HPV 16 and HPV 18 using the polymerase chain reaction with type specific internal probes.

Results-There were seven verrucous carcinomas, and 11 well, 17 moderately, and six poorly differentiated squamous cell carcinomas. Six of the $41(15 \%)$ patients had penile carcinoma containing HPV 16 or HPV 18 DNA, or both, with HPV 16 found in four (10\%) and HPV 18 in four $(10 \%)$. The mean ages of HPV positive and HPV negative groups of patients were 68.5 and 57.6 years, respectively $(p<0 \cdot 05)$. None of the seven verrucous and 11 well differentiated squamous cell carcinomas was positive for HPV. The mean age of patients who had these carcinomas was 52.4 years. As a group, these low grade carcinomas occurred in patients younger by more than a decade than those who had carcinomas of the higher grades (mean age 64.4 years; $p$ $<0 \cdot 01$ ).
\end{abstract}

Conclusions-Penile carcinomas had much lower rates of infection by HPV 16 or HPV 18 than cervical carcinomas in this Hong Kong population. Based on our findings and on data collated from published findings, it is concluded that penile verrucous carcinomas are not associated with HPV 16 and HPV 18. The overall low prevalence of HPV 16 and HPV 18 in penile carcinomas suggests that other HPV types might be important in the pathogenesis of these tumours.

Department of Pathology, University of Hong Kong, Hong Kong

KW Chan

KY

A C L Chan

A C L

G Srivastava

Correspondence to: Dr KW Chan Accepted for publication 28 February 1994 types. ${ }^{1}$ Specific types of HPV, most commonly type 16 (HPV 16) and less commonly type 18 (HPV 18), have been implicated in the aetiology of cervical squamous cell carci- nomas. ${ }^{2}$ Although genital HPV infections are sexually transmitted, information on the association between HPV and penile carcinomas has seldom been collected, probably because of the low incidence of these tumours compared with cervical carcinomas. ${ }^{3}$

In the present study, we used the highly sensitive and specific polymerase chain reaction (PCR) to define the association between HPV 16 and HPV 18 with penile carcinomas. Our findings are compared with relevant data reported in other countries and the incidence of HPV 16 and 18 in invasive cervical carcinomas of the local population. To the best of our knowledge, this study is the first large series of penile carcinomas in Chinese patients examined for HPV by this highly specific and sensitive DNA detection method.

\section{Methods}

Cases of verrucous and squamous cell carcinoma of the penis from 1974-1992 were retrieved from the archives of the Department of Pathology at Queen Mary Hospital. Only surgical specimens containing the primary tumours were selected. The slides were reviewed and the diagnosis confirmed by two pathologists (KY Lam and ACL Chan). The tumours were classified into verrucous carcinoma, and well, moderately, and poorly differentiated squamous cell carcinomas. The criteria used for differentiating verrucous carcinoma were a densely keratinised surface and a sharply delineated deep margin with a more expansive margin composed of bulbous downgrowths of well differentiated keratinising squamous epithelium. ${ }^{4}$ One representative block from each haematoxylin and eosin stained specimen was sectioned and subjected to PCR analysis.

The oligonucleotide primers used in the PCR for HPV 16 were complementary to the upstream regulatory region of the viral DNA, ${ }^{5}$ while for HPV 18 they were complementary to sequences in the E6 region. ${ }^{6}$ These regions were chosen because they are almost always retained even if the HPV DNA is integrated in tumours. The sequences of the primers used are shown in table 1.

In each case, a $16 \mu \mathrm{m}$ thick section was cut from the paraffin wax embedded tumour tissue and waxed with sequential washes of xylene, 95\% ethanol, and absolute ethanol and then vacuum dried. The section was resuspended in $50 \mu \mathrm{l}$ of water, boiled for 20 minutes, and snap cooled on ice to release and denature the DNA before PCR analysis. 
Table 1 Sequence of oligonucleotide primers

\begin{tabular}{lll}
\hline HPV type & Sequence $\left(5^{\prime}->3^{\prime}\right)$ & Size of amplified product (base pairs) \\
\hline HPV 16 & A: AAG GCC AAC TAA ATG & 228 \\
& TCAC (19-mers) & \\
& B: GCG GAT CCT GTC TGC & \\
& TTT TAT ACT AA (26-mers) & \\
& Probe: TTT CGG TTC AAC CGA & \\
TTT CGG (21-mers) & \\
HPV 18 & A: ACC TTA ATG AAA AAC & 100 \\
& GAC GA (20-mers) \\
& B: CGT CGT TGG AGT CGT & \\
& TCC TG (20-mers) \\
& Probe: TAG CTG GGC ACT ATA \\
& GAG GC (20-mers) & \\
\hline
\end{tabular}

Amplification of the HPV 16 or HPV 18 targeted DNA sequences was carried out for 40 cycles using a GeneAmp PCR Core Reagent kit (Perkin Elmer Cetus, Connecticut, USA) and programmable thermal controller (MJ Research, Inc., Massachusetts, USA), with $37^{\circ} \mathrm{C}$ as the annealing temperature (for two minutes). The amplified products were analysed by gel electrophoresis in $4 \%$ NuSieve 3:1 agarose (FMC Bioproducts, Maine, USA), followed by ethidium bromide staining. The specificity of the amplified product was confirmed by Southern blotting of the gel, followed by probing of the membrane with ${ }^{32} \mathrm{P}$-labelled internal probes specific for the amplified HPV 16 or HPV 18 DNA. The hybridisation and washing conditions were identical with those previously reported by our laboratory. ${ }^{7}$

DNA extracted from CaSki and HeLa cell lines, obtained from the American Tissue Type Culture Collection, were used as positive controls for HPV 16 and HPV 18, respectively. DNA extracted from paraffin wax sections of rat kidney was used as a negative control in each set of PCR.

The following additional precautions were taken to prevent possible cross-contamination between the samples during the PCR procedure: (1) sections were cut using different disposable microtome blades for each sample, and negative control blocks were cut between the cases under study; (2) all the manipulations preceding PCR analysis were performed in a separate room away from the PCR machine; and (3) the PCR products were analysed in a separate room. As a positive amplification control, primers (PC03 and PC04) specific for human $\beta$ globin gene were used in PCR analysis for all the samples studied, as described by Saiki et al, ${ }^{8}$ using $56^{\circ} \mathrm{C}$ as the annealing temperature (for two minutes). The ${ }^{32} \mathrm{P}$-labelled oligomeric primer RS06 was

Table 2 Penile carcinoma and HPV types 16 and 18

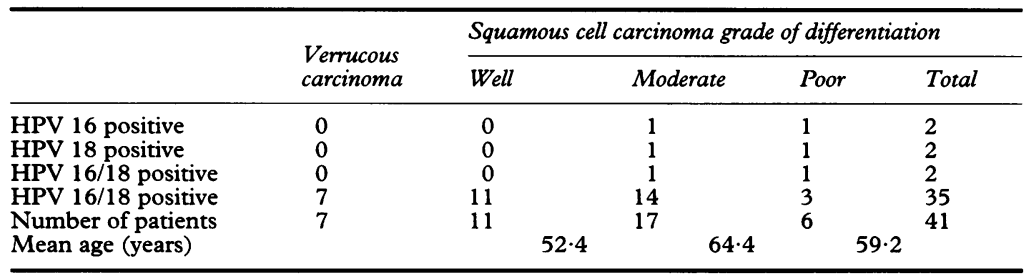

used as an internal probe to confirm the specificity of the amplified product (110 base pairs).

Statistical analysis was performed using Fisher's exact and Student's $t$ tests.

\section{Results}

A total of 43 patients were identified as having verrucous or squamous cell carcinoma of the penis in the period 1974 to 1992 . The block from one specimen showed no PCR amplification of the $\beta$ globin gene. It was concluded that the quality of DNA in this specimen was poor and was thus excluded from the study. Another specimen was excluded because insufficient DNA was left to complete the $\beta$ globin PCR. The results of PCR amplification of HPV on 41 specimens were subjected to statistical analysis.

The age of the 41 patients ranged from 29 to 84 years. The mean and median ages of the 41 patients were 59 years. The only nonChinese patient encountered was a 29 year old Vietnamese man. When patients were divided into two groups according to the histological grades of their tumours with their mean ages compared (table 2), the difference was highly significant $(\mathrm{p}<0.01, t$ test).

Of the 41 tumours selected, six were positive for either HPV 16 or HPV 18, or both. Table 2 shows the distribution of HPV 16 and HPV 18 in the tumours according to histological types and grades. The six patients who had HPV in their tumours had a mean age of 68.5 years. This was significantly higher than the 57.6 years, the mean age of the group of patients who had no HPV in their tumours ( $p$ $<0.05$; $t$ test). When the mean age of HPV positive patients were compared with that of HPV negative patients having the same histological grades of tumours, there was no significant difference ( $p>0 \cdot 10 ; t$ test).

\section{Discussion}

Different methods have been used to detect type specific HPV in penile carcinomas. These include Southern blotting, ${ }^{910}$ in situ hybridisation, ${ }^{4112}$ and PCR. ${ }^{12-14}$ Among these, PCR seems to be superior because of its high sensitivity. Varma $e t a l^{12}$ recently applied both in situ hybridisation and PCR to study the incidence of HPV types in penile carcinomas. The concordance rate of the results obtained by both techniques were high. They found two instances of false negative results of in situ hybridisation in resection specimens and thought that some problems in obtaining optimally preserved tissue in archival resection specimens were responsible. PCR was able to detect the virus in one of the two resection specimens which gave false negative results by in situ hybridisation. PCR alone was therefore chosen for the purpose of the present study.

The prevalence of HPV in the uterine cervix and the strong association between certain HPV types and cervical carcinomas is well documented. ${ }^{267} \mathrm{~A}$ recent study of 45 
invasive carcinomas of the uterine cervix performed in our laboratory using the same PCR technique $^{7}$ showed that $69 \%$ and $44 \%$ of the tumours were positive for HPV 16 and HPV 18 , respectively, while $82 \%$ of the tumours were positive for at least one of these two types of HPV. The $10 \%$ incidence of HPV types 16 and 18 in penile carcinomas was thus much lower than the corresponding figures found in cervical carcinomas in Hong Kong ( $p<0.001$; Fisher's exact probability test). The findings suggested that HPV types 16 and 18 were unlikely to have a strong association with penile carcinomas in Hong Kong, and that the aetiological factors for penile carcinomas might be different from those for cervical carcinomas.

Table 3 summarises the reported incidences of HPV types 16 and 18 in penile carcinomas. The data we had listed were from Brazil, ${ }^{9-11}$ the USA, ${ }^{42-14}$ and Australia. ${ }^{11}$ Brazil has a high incidence of penile carcinoma compared with other countries in the world. Hong Kong has a low incidence of penile squamous cell carcinoma comparable with that in Australia and the USA. Our results showed a generally lower incidence of HPV 16 and a slightly higher incidence of HPV 18 in penile carcinomas than those found elsewhere.

In our study none of the well differentiated or verrucous carcinomas was positive for HPV 16 or HPV 18. It is worth noting that Masih et $a l^{4}$ in the USA were unable to find HPV types $6,11,16,18$, and 31 in any of their 10 cases of penile verrucous carcinomas. Kiyabu and colleagues ${ }^{13}$ studied two and three cases of nonverrucous and verrucous penile carcinomas, respectively. HPV 16 was identified in the two non-verrucous, but not in the verrucous, carcinomas. Their five cases were uniformly negative for HPV 18. Higgins $e t a l^{11}$ similarly could not detect HPV in all the five verrucous carcinomas studied, but HPV RNA was detected in one of eight well differentiated, and nine of 18 higher grade carcinomas, studied. There were no examples of verrucous carcinoma in the series of Wiener $e t a l^{14}$ who found one of the four well differentiated carcinomas positive for HPV 16. Of 17 penile carcinomas of higher grades they studied, five and one were positive for HPV 16 and HPV 18, respectively. None of the four penile verrucous carcinomas studied by Varma et $a l^{12}$ was positive for HPV 16 or HPV 18. In the study by Villa

Table 3 Summary of published data on penile verrucous and squamous cell carcinoma associated HPV 16 and HPV 18 infections

\begin{tabular}{lllll}
\hline Source & Method & Country & HPV 16 & HPV 18 \\
\hline Villa and Lopes, 1986 & Southern blot & Brazil & $0 / 18(0 \%)$ & $7 / 18(39 \%)$ \\
McCance, et al, 1986 & Southern blot & Brazil & $26 / 53(49 \%)$ & $5 / 53(9 \%)$ \\
Kiyabu, al, 1989 & PCR & USA & $2 / 5(40 \%)$ & $0 / 5(0 \%)$ \\
Masih, et al, 1992 & ISH & USA & $0 / 10(0 \%)$ & $0 / 10(0 \%)$ \\
Varma, et al, 1992 & PCR, ISH & USA & $12 / 18(67 \%)$ & $0 / 18(0 \%)$ \\
Higgins, et al, 1992 & ISH & Australia & $7 / 22(32 \%)$ & $0 / 22(0 \%)$ \\
& ISH & Brazil & $2 / 9(22 \%)$ & $0 / 9(0 \%)$ \\
Wiener, et al, 1992 & PCR & USA & $6 / 21(29 \%)$ & $1 / 21(5 \%)$ \\
Present study & PCR & Hong Kong & $\mathbf{4} / 41(10 \%)$ & $\mathbf{4} / \mathbf{4 1}(10 \%)$ \\
\hline
\end{tabular}

ISH = in situ hybridisation; $\mathrm{PCR}=$ polymerase chain reaction. and Lopes, ${ }^{9}$ there was one penile verrucous carcinoma which was negative for HPV 16 and HPV 18. McCance et al ${ }^{10}$ did not subclassify the tumours in their series. Our study showed that HPV DNA was present in three $(50 \%)$ of the poorly differentiated, and three (18\%) moderately differentiated squamous cell carcinomas. Other studies have found a high incidence of HPV 16 in grade 3 penile intraepithelial neoplasia. ${ }^{11}$

If HPV types 16 or 18 were responsible for neoplastic transformation of penile carcinomas, irrespective of histological types and grades, it seemed unlikely that viral DNA should disappear from the better differentiated tumours while being retained in the less differentiated ones. The more likely explanation is that different aetiological effectors were operative in these different groups of penile carcinomas. To support this view we found the less differentiated and HPV positive penile carcinomas in our series occurring in a significantly older age group of patients than those better differentiated tumours. Based on the evidence accumulated so far, we concluded that penile verrucous carcinomas were rarely, if ever, associated with HPV types 16 and 18.

In summary, our study shows that HPV 16 and HPV 18 each infected $10 \%$ of penile carcinomas in Hong Kong. Infection by both HPV types occurred in 5\% of penile carcinomas. The incidence was very much lower than that found in cervical carcinomas studied by our laboratory. The older age of patients who had penile carcinomas of higher grades and the exclusive occurrence of HPV types 16 and 18 in this group of tumours suggest that the better differentiated tumours, unlike the higher grade tumours, were not associated with HPV types 16 and 18. The absence of HPV types 16 and 18 in penile verrucous carcinoma in our study and several others suggested that there was no association between these HPV types and penile verrucous carcinoma. Further investigations are required to find out whether penile carcinomas are associated with other HPV types.

This work was supported by a grant from The University of Hong Kong (RGC research grant 337/046/0019).

1 de Villiers EM. Heterogeneity of the human papillomavirus group. F Virol 1989;63:4898-903.

2 McCance DJ. Human papillomavirus (HPV) infections in the aetiology of cervical cancer. Cancer Surv 1988;7: 499-506.

3 Muir C, Waterhouse J, Mack T, Powell J, Whelan S, eds. Cancer incidence in five continents. Volume V. IARC Scientific Publication No 88. Lyon: International Agency Scientific Publication No 88. Lyon

4 Masih AS, Stoler MH, Farrow GM, Wooldridge TN, Johansson SL. Penile verrucous carcinoma: A clinicopathologic, human papillomavirus typing and flow cytometric analysis. Mod Pathol 1992;5:48-55.

5 Tidy JA, Vousden KH, Farrell PJ. Relation between infection with a subtype of HPV 16 and cervical neoplasia. Lancet 1989;i:1225-7.

6 Young LS, Bevan IS, Johnson MA, et al. The polymerase chain reaction: a new epidemiological tool for investigating cervical human papillomavirus infection. $\mathrm{Br}$ Med $\mathcal{f}$ 1989;298:14-8.

7 Ip EWK, Collins RJ, Srivastava G, Cheung ANY, Wong LC. Human papillomavirus and its prognostic significance in invasive carcinoma of cervix in young patients. cance in invasive carcinoma of cervix

8 Saiki RK, Scharf S, Falloona F, et al. Enzymatic amplification of $\beta$-globin genomic sequences and restriction site analysis for diagnosis of sickle cell anemia. Science 1985;230:1350-4. 
9 Villa LL, Lopes A. Human papillomavirus DNA sequences in penile carcinomas in Brazil. Int $\mathcal{F}$ Cancer 1986;37:853-5.

10 McCance DJ, Kalche A, Ashdown K, et al. Human papillomavirus types 16 and 18 in carcinomas of the penis from Brazil. Int $\mathcal{Y}$ Cancer 1986;37:55-9.

11 Higgins GD, Uzelin DM, Philips GE, Villa LL, Burrell CJ Differing prevalence of human papillomavirus RNA in penile dysplasias and carcinomas may reflect differing etiologies. Am 7 Clin Pathol 1992;97:272-8.

12 Varma VA, Sanchez-Lanier M, Unger ER, et al. Association of human papillomavirus with penile carcinoma: A study using polymerase chain reaction and in situ hybridization. Hum Pathol 1991;22: 908-13.

13 Kiyabu MT, Shibata D, Arnheim N, Martin WJ, Fitzgibbons PL. Detection of human papillomavirus in formalin-fixed, invasive squamous carcinomas using the polymerase chain reaction. Am f Surg Pathol 1989;13: $221-4$.

14 Wiener JS, Effert PJ, Humphrey PA, Yu L, Liu ET, Walther PJ. Prevalence of human papillomavirus types 16 and 18 in squamous-cell carcinoma of the penis: A 16 and 18 in squamous-cell carcinoma of the pestive analysis of primary and metastatic lesions by differential polymerase chain reaction. Int $₹$ Cancer 1992;50:694-701. 